\title{
Characterization of Advanced High-Energy Density Li-S Batteries by FE- AEM, SEM/EDS X-ray Spectral Imaging and Feature Sizing/Chemical Typing Techniques
}

\author{
V. P. Oleshko ${ }^{1}$, C. Scordilis-Kelley ${ }^{1}$, A. Xiao ${ }^{1}$, J. Affinito $^{1}$, Y. Talyossef ${ }^{2}$, R. Elazari ${ }^{2}$, \\ Y. Grinblat ${ }^{2}$ and D. Aurbach ${ }^{2}$ \\ ${ }^{1}$ Sion Power, Tucson AZ 85756-7129 and ${ }^{2}$ Bar-Ilan University Ramat Gan, 52900 Israel
}

Environmentally clean technologies for sustainable energy generation and use are continuously demanding a next generation of long-cycle life high-energy density battery systems, which have higher capacity than existing electrochemical Li-ion and Li-ion/polymer batteries. A light-weight rechargeable cell based on the Li/elemental $\mathrm{S}$ redox couple has a highest theoretical specific capacity of $1670 \mathrm{mAh} / \mathrm{g}$ of active material and a specific energy of $2600 \mathrm{Wh} / \mathrm{kg}$, assuming complete reaction $2 \mathrm{Li}^{\mathrm{O}}+\mathrm{S}^{\mathrm{o}} \rightarrow \mathrm{Li}_{2} \mathrm{~S}$ [1]. This Sion Li-S battery type utilizing liquid electrolytes with a specific energy $350 \mathrm{Wh} / \mathrm{kg}$ is superior to commercial type Li-ion batteries delivering specific energies $64-108 \mathrm{Wh} / \mathrm{kg}$ that demonstrated in 2007-2008 by setting two unofficial world endurance records for a flight by an unmanned Zephyr-6 aircraft (QinetiQ, UK) [2]. To allow a broader range of emerging applications such as electrical vehicles, performance of Li-S batteries at continuous high current discharge will be further improved via novel design of cells and electrode materials. Rechargeability and utilization of active material limited by the insulating nature of sulfur and $\mathrm{Li}_{2} \mathrm{~S}$ and losses of active material in the form of insoluble polysulfides (PS) should be enhanced. Here we report results on combined structural and analytical characterization of commercially available unprotected Li foil anodes and sulfur-carbon cathodes down to the atomic scale by FE-AEM, FESEM, X-ray spectral imaging (SI) and feature sizing/chemical typing techniques performed to address several key problems for Li-S cells.

3D-morphology, crystallography and elemental distributions over porous sulfur-carbon cathodes (FIG. 1). Sulfur-carbon cathodes exhibited extended aggregated and porous structures. Lowvoltage high-resolution FESEM enabled to characterize in detail surface morphology of faceted sulfur microcrystals, aggregated carbons and individual carbon particles down to 5-10 nm. EDS X-ray SI using drift compensation followed by data multivariate statistical analysis indicated an appearance of sulfur-enriched segregations on the scale of 10-15 $\mu \mathrm{m}$. FESEM and HRTEM combined with SAED and EDS revealed crystalline structures and local compositions of sulfur and PS particles and $\mathrm{Li}_{2} \mathrm{~S}$ nanocrystallites formed under cycling.

Morphological and chemical classification of a cycled Li anode surface by automated feature sizing/chemical typing analysis (FIG. 2). The anode was fabricated using a $50.8 \mu \mathrm{m}$-thick commercially available unprotected Li foil. Thermo NORAN System SIX feature sizing and chemical typing analysis module provides flexible means to measure the surface feature size/shapes and count their number in selected anode areas from SE or BSE images using various morphological parameters (area, circularity, aspect ratio, etc.), filters and sieves to tailor desired analysis. Feature sizing analysis data evidently pointed to significant morphological changes of the anode surface under chosen cycling conditions. In addition, chemical typing allowed classifying of analyzed surface areas vs. contents of $\mathrm{C}, \mathrm{O}, \mathrm{N}, \mathrm{F}$ and $\mathrm{S}$ by matching their quantitative chemical composition to a database of chemical types created in a chemical library.

References

[1] D. Marmorstein, et al., J. Power Sources 89 (2000) 219. [2] http://news.bbc.co.uk/go /pr/fr//2/hi/science/nature/7577493.stm. Published: 2008/08/24 04:21:42 GMT. 

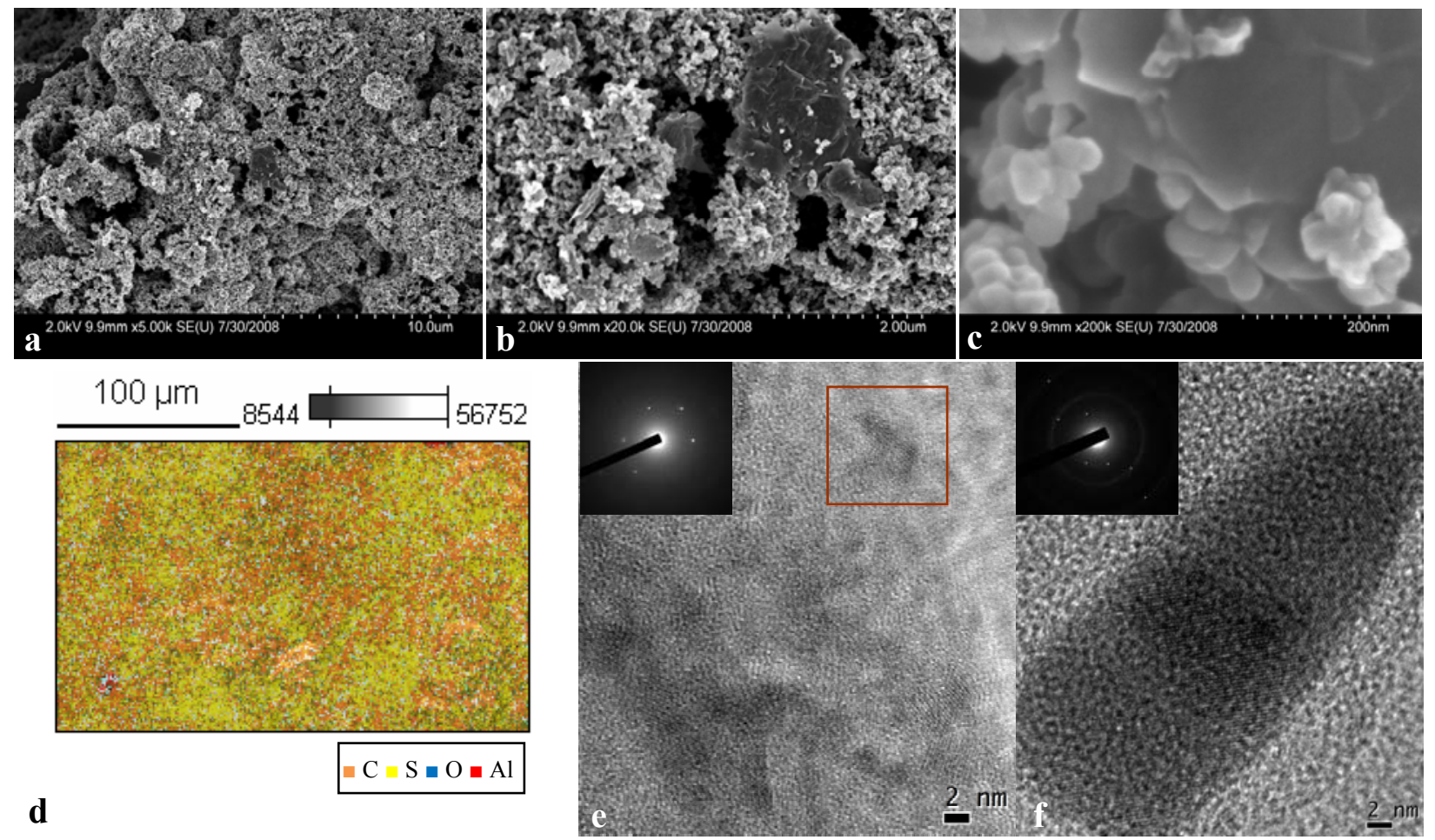

FIG. 1. Sulfur-carbon cathode, low-voltage HR-FESEM, (a) aggregated carbon and porous structures, (b) sulfur microcrystals, (c) 10-40 nm carbon particles at a sulfur-carbon interface. Xray SI, (d) composite overlay of CK, SK, OK and AlK X-ray maps showing 10-15 $\mu \mathrm{m}$ sulfur segregations. HRTEM, (e) a nanocrystallite (in a square) near the edge of a sulfur particle and its SAED pattern (inset) (f) a $\mathrm{Li}_{2} \mathrm{~S}$ crystal formed during cathode cycling and its SAED pattern (inset).

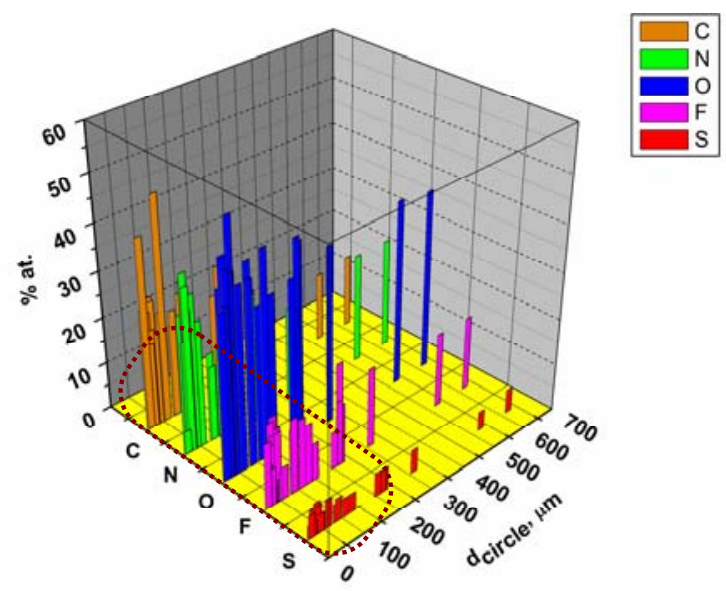

FIG. 2. Morphology and chemical composition classification of a charged unprotected Li foil anode surface by an automated NORAN SIX feature sizing analysis module. 3D-bar plot presents distributions of the analyzed surface areas by size (mean equivalent diameter is $98 \mu \mathrm{m}$, st. dev. is $130 \mu \mathrm{m}$; median is $39 \mu \mathrm{m}$ and range is $610 \mu \mathrm{m}$ ) and composition (contents of C, mean is $22.2 \%$ at., st. dev. is 7.0 ; $\mathrm{N}$, mean is $23.3 \%$ at., st. dev. is 9.1 ; O, mean is $39.3 \%$ at., st. dev. is 4.2; F, mean is $10.2 \%$ at., st. dev. is 6.2 and $\mathrm{S}$, mean is $3.5 \%$ at., st. dev. is 2.0 , respectively). Most significant morphological and compositional changes occurred within the area shown by a dash line. 